\title{
Controlled Release of Alendronate from Nitrogen-Doped Mesoporous Carbon
}

\section{Dipendu Saha ${ }^{1, *}$, Amanda Spurri ${ }^{1}$, Jihua Chen ${ }^{2}$, Dale K. Hensley ${ }^{2}$}

1. Department of Chemical Engineering, Widener University One University Place, Chester, PA 19013, USA,

2. Center for Nanophase Materials Sciences, Oak Ridge National Laboratory, Oak Ridge, TN 37831, USA,

*Corresponding author's E-mail: dsaha@mail.widener.edu (D. Saha), Phone: +1 6104994056 , Fax: 6104994059

To be submitted to Microporous and Mesoporous Materials (short communication) 


\begin{abstract}
We have synthesized a nitrogen doped mesoporous carbon with the BET surface area of $1066 \mathrm{~m}^{2} / \mathrm{g}$, total pore volume $0.6 \mathrm{~cm}^{3} / \mathrm{g}$ and nitrogen content of $0.5 \%$. Total alendronate adsorption in this carbon was $\sim 5 \%$. The release experiments were designed in four different media with sequential $\mathrm{pH}$ values of $1.2,4.5,6.8$ and 7.4 for $3,1,3$ and 5 hours, respectively and at $37^{\circ} \mathrm{C}$ to imitate the physiological conditions of stomach, duodenum, small intestine and colon, respectively. Release of the drug demonstrated a controlled fashion; only $20 \%$ of the drug was released in the media with $\mathrm{pH}=1.2$, whereas $64 \%$ of the drug was released in $\mathrm{pH}=7.4$. This is in contrary to pure alendronate that was completely dissolved within 30 minutes in the first release media $(\mathrm{pH}=1.2)$ only. The relatively larger uptake of alendronate in this carbon and its sustained fashion of release can be attributed to the hydrogen bonding between the drug and the nitrogen functionalities on carbon surface. Based on this result, it can be inferred that this formulation may lower the side effects of oral delivery of alendronate.
\end{abstract}

Keywords: Nitrogen doping, mesoporous carbon, alendronate, osteoporosis, controlled release 


\section{Introduction}

Bisphosphonates are one type of synthetically produced drugs that are structurally similar to that of naturally occurring pyrophosphates. Alendronate and Zoledronate are two typical bisphosphonates, in which alendronate has the most common application in bone related problems. Alendronate is an aminobisphosphonate (figure 1) and it can suppress the activity of osteoclast cells thereby lowering the osteoclast-mediated bone resorption [1-5]. In addition to that, in-vivo and in-vitro studies proposed that bisphosphonates, like alendronate could possess osteo-stimulative properties [6,7] and mineralize nodules to help early osteoblastogenesis [8,9]. Recent studies demonstrated a possible relation between systematics usages of alendronate and avascular osteonecrosis of the jaw [10,11]. Alendronate has also shown a positive role in the treatment of intra-bony defects in patients with chronic periodontitis [12] or bone metastasis $[13,14]$. It is a well-prescribed medicine for osteoporosis, like post-menopausal osteoporosis, that causes bone weakening and easy fracturing of bones. Despite helpful effects in bone-related abnormalities, there are some severe side effects of this drug, especially for oral delivery. It causes severe burning in the esophagus followed by other gastro-intestinal problems [15]. It has been suggested that the patient should follow a strict regulation of staying upright for at least 30 minutes after taking this medication to minimize such effect. Besides that, it has very low bioavailability $(\sim 1 \%)$ owing to poor absorption in the intestine [16,17] and rapid clearance from blood [18]. In order to enhance its total uptake, a higher dose is usually prescribed that cumulatively worsens the side effects.

Several passive formulations for alendronate delivery have been developed, but majority of them were focused in bio-ceramics for direct or local delivery towards rapid bone healing. Calcium phosphate or hydroxyapatite-based local delivery system for controlled release of alendronate has been reported successfully. The drug was directly loaded onto the bulk ceramic [19-21], functionalized with gelatin [22] or formulated with calcium phosphate and loaded into porous titanium [23]. Besides phosphates, silica based materials were quite popularly employed towards local delivery of alendronate. Ordered mesoporous silica [24,25] and aminopropyl [26] or phosphorous [27] doped silica were successfully developed for alendronate release. Different biocompatible polymeric materials were also developed for direct release of alendronate. Poly (lactic-co-glycolic acid) (PLGA) loaded hydroxyapatite microspheres were developed for control 
release of alendronate for bone repair [28]; pure PLGA/alendronate formulations were also employed for dental applications [29]. Polyethylene glycol (PEG)-dendrimer and $\mathrm{H}_{2} \mathrm{~N}-\mathrm{PEG}$ dendrimer- $(\mathrm{COOH})_{4}$ based polymeric systems were successfully reported as combined carriers of alendronate and paclitaxel [30]. Different types of bisphosphonate carriers are discussed in the review [31]. In order to enhance the oral bioavailability of alendronate, Han et al. [32] employed chitosan-coated liposomes with $200 \mathrm{~nm}$ size that enhanced the cellular uptake of the drug in Caco- 2 cells. These drug carriers increased the oral bioavailability by 2.6 times compared to free drug in rat-based animal model study.

In today's perspective, carbon-based drug carriers have gained lot of interests in biomedical platforms. Traditionally, activated carbon material was employed as a remedy for drug overdose or poison arrester upon accidental indigestion. Different carbon materials that have already demonstrated their potential role in controlled drug delivery are carbon nanotube, carbon dots or mesoporous carbons. Especially, mesoporous carbons have demonstrated a successful role in both bulk and nano forms [33,34]. Different research groups, including ours reported the control release of multitude of drugs from mesoporous carbon-based materials, including captopril [35,36], furosemide [36], ranitidine hydrochloride [37], ibuprofen [37], antipyrine [38,39] indomethacin [39] and others. Our recent study confirmed the biocompatibility of mesoporous carbon materials in terms of cytotoxicity with HeLa cell, cell viability with fibroblast cells, hemolysis, and protein adsorption results [40]. Our recent study also confirmed the benign nature of mesoporous carbons compared to nano-carbons through the measurement of reactive oxygen generation, Adenosine Triphosphate (ATP) depletion and cellular uptake through TEM visualization [41]. Motivated by the potential role of mesoporous carbons in controlled drug delivery, we have employed nitrogen doped mesoporous carbon in the controlled release of alendronate towards oral delivery.

\section{Experimental}

\subsection{Synthesis of nitrogen doped mesoporous carbon}

Nitrogen doped mesoporous carbons were synthesized from the modified procedures reported by Wei et al. [42] Typically, 5 g resorcinol and 5 g Pluronic F127 were dissolved in 30 $\mathrm{cm}^{3}$ solvent composed of 1:1 volumetric ratio of water and ethanol along with $0.6 \mathrm{~cm}^{3} 36 \% \mathrm{HCl}$. Upon mixing, $5 \mathrm{~cm}^{3} 37 \%$ formaldehyde solution was added as cross-linking agent and stirred for 
3 hours. After that, $5 \mathrm{~g}$ dicyandiamide was added to the mixture and stirred for 48 hours. Then the mixture was separated from the solvent and cured at $200{ }^{\circ} \mathrm{C}$ for two hours in air and then put to carbonization in a porcelain boat in a tube furnace. In the course of carbonization, it was heated to $400{ }^{\circ} \mathrm{C}$ in $1{ }^{\circ} \mathrm{C} / \mathrm{min}$ followed by $1000{ }^{\circ} \mathrm{C}$ at $2{ }^{\circ} \mathrm{C} / \mathrm{min}$ and then cooled down to ambient temperature. In order to further activate the carbonized material, it is mixed solid $\mathrm{KOH}$ in 1:3 ratio and heated in nitrogen atmosphere up to $1000{ }^{\circ} \mathrm{C}$ in $5{ }^{\circ} \mathrm{C} / \mathrm{min}$ and then cooled down to ambient temperature. All the heating and cooling operations are performed in nitrogen atmosphere. The activated carbon material was washed with DI water several times and then filtered and dried before any characterization.

\subsection{Materials characterization}

The mesoporous carbon was characterized with pore size distribution by $\mathrm{N}_{2}$ adsorptiondesorption at liquid nitrogen temperature $(77 \mathrm{~K})$ and $\mathrm{CO}_{2}$ adsorption at $273 \mathrm{~K}$, electron microscopy by TEM and SEM-EDX and thermal stability by thermogravimetric analysis (TGA). The $\mathrm{N}_{2}$ and $\mathrm{CO}_{2}$ adsorption-desorption measurements were performed in Quantachrome's Autosorb-iQ instrument. To maintain $273 \mathrm{~K}$ for $\mathrm{CO}_{2}$ adsorption, a Julabo ${ }^{\oplus}$ temperature controller was employed with 1:1 mixture of water and propylene glycol as chilling fluid. $\mathrm{N}_{2}$ adsorption at $77 \mathrm{~K}$ was employed to calculate Brunauer-Emmett-Teller (BET) surface area, whereas both $\mathrm{N}_{2}$ and $\mathrm{CO}_{2}$ adsorption were used to calculate pore size distribution by non-local density function theory (NLDFT) in instrument's built-in software. Thermogravimetric Analysis (TGA) was performed in TA instruments' SDT Q600 instrument. TEM images were captured in Carl Zeiss Libra 120 TEM operating at $120 \mathrm{kV}$. The scanning electron microscopy (SEM) imaging was performed in a Carl Zeiss Merlin SEM equipped with EDX facility.

\subsection{Alendronate loading and release}

In order to load (adsorb) alendronate onto nitrogen doped mesoporous carbon, $0.1 \mathrm{~g}$ of pure alendronate is dissolved onto $10 \mathrm{~cm}^{3}$ DI water adjusted with $\mathrm{pH}$ of 1.2 by $\mathrm{HCl}$ and then, 0.5 $\mathrm{g}$ of pure mesoporous carbon was added and stirred overnight. After that, the carbon is separated from the solution by filtration and quickly washed with DI water to remove alendronate that might have accumulated outside from the solution. The carbon was dried in oven at $100{ }^{\circ} \mathrm{C}$ overnight and used thereafter. The drug-loading amount was determined by comparing 
thermogravimetric analysis (TGA) data of pure drug, pure carbon and drug-loaded carbons. During TGA study, all the materials were heated upto $105{ }^{\circ} \mathrm{C}$ for 3 minutes to eliminate effect of pre-loaded moisture or $\mathrm{HCl}$.

In order to investigate the controlled release of alendronate from mesoporous carbons, all the release experiments were conducted for $0.1 \mathrm{~g}$ of alendronate loaded carbon in $50 \mathrm{~mL}$ release media at $37{ }^{\circ} \mathrm{C}$. The $\mathrm{pH}$ and exposure time of the release media were sequentially set to $1.2,4.5$, 6.8 and 7.4 for $3,1,3$, and 5 hours, respectively to mimic the physiological conditions of stomach, duodenum, small intestine and colon, respectively [39]. The medium with $\mathrm{pH}=1.2$ (simulated gastric fluid, SGF) was composed of $\mathrm{HCl}$ and $1 \% \mathrm{NaCl}$ without any enzyme. Rest of the $\mathrm{pH}$ was set by $\mathrm{HCl}$ adjustment only. At the specific time intervals, $2.5 \mathrm{~cm}^{3}$ of the release medium was withdrawn and replenished immediately with fresh release media. The collected sample was preserved for further analysis. After completing release medium of one $\mathrm{pH}$, the carbon is separated, dried and inserted onto the next medium with corrected weight. All the runs were repeated thrice and the average values were reported with standard deviation.

Since alendronate is not UV active, it was derivatized for UV active component with ninhydrin according to a modified procedure reported by Sultana et al. [43] Typically, a reagent mixture was prepared by mixing $0.13 \mathrm{~g}$ ninhydrin and $0.085 \mathrm{~g} \mathrm{NaHCO}_{3}$ in $50 \mathrm{~cm}^{3}$ methanol and $10 \mathrm{~cm}^{3}$ DI water. $2.5 \mathrm{~cm}^{3}$ alendronate solution was mixed with 60 microliters of $10 \mathrm{M} \mathrm{NaOH}$ solution and then added with $3 \mathrm{~cm}^{3}$ reagent mixture followed by heating at $100{ }^{\circ} \mathrm{C}$ for 30 minutes. After that, the mixture was cooled, mixed with DI water to make the total volume upto $7.5 \mathrm{~cm}^{3}$ and analyzed in UV spectroscope. To study the release alendronate, a tablet of pure alendronate $(0.1 \mathrm{~g})$ was prepared in a machine press and the release experiments were performed in the same fashion. To collect the samples for pure alendronate, 13 microliters of release medium was mixed with $2.5 \mathrm{~cm}^{3}$ of stock solution and rest of the analysis protocols were same as that alendronate loaded carbons. For UV spectroscopic measurements, a calibration plot was made with the alendronate concentrations of $60,50,40,30,20$, and 10 ppm with their corresponding absorbance values at the wavelength of $420 \mathrm{~nm}$. The proposed reaction mechanism between alendronate and ninhydrin was similar to that of primary amine detection by ninhydrin and is given by 


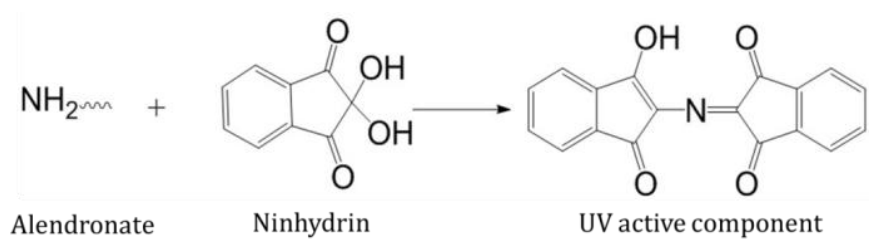

\section{Results and Discussions}

\subsection{Materials characteristics}

Nitrogen adsorption-desorption plot at $77 \mathrm{~K}$ of the carbon is shown in the inset of figure 2. It is of type IV according to IUPAC classifications. The hysteresis loops is originated due to the condensation of the nitrogen in the mesopores. Brunauer-Emmett-Teller (BET) surface area of this carbon is $1066 \mathrm{~m}^{2} / \mathrm{g}$. Figure 2 represents the combined pore size distribution calculated by NLDFT theory from the nitrogen and carbon dioxide adsorption plots. In the narrow micropore region, it has the pore width in the range of 3.5, 5 and $7.85 \AA$. It has a distributed mesoporosity within the range of 20 to $90 \AA$. Despite similar protocol of synthesizing pure mesoporous carbons demonstrated a clear mesopore peak within the range of 50 to $60 \AA$ in our past studies, such distorted mesoporosity might have been caused by the presence of dicyandiamide in the reaction mixture. The total pore volume of the carbons is $0.6 \mathrm{~cm}^{3} / \mathrm{g}$. Thermogravimetric analysis (TGA) of this material (figure S1, supporting information) demonstrated about $8 \%$ mass loss when heated in nitrogen atmosphere upto $1000{ }^{\circ} \mathrm{C}$, which might have been caused by the dissociation of the oxygen and nitrogen containing functional groups from the carbon surface. SEM and TEM images (figure 3) reveal that the particles are irregular in shape and have a wide size distribution. Smaller particles are in the range of 1 to $1.5 \mu \mathrm{m}$, whereas the larger particles are within $200 \mu \mathrm{m}$. They have a rough surface topography and no ordered porosity is observed in high magnification SEM or TEM images. Figure 3(e) and (f) show the Energy Dispersive X-ray (EDX) image of the carbon; magenta and green dots show the presence of carbon and nitrogen atoms, respectively. A separate study on X-ray photoelectron spectroscopy (XPS) (figure S2, supporting information) revealed that this mesoporous carbon has about $0.5 \%$ nitrogen. 


\subsection{Alendronate loading and release}

Alendronate loading amount was $\sim 5 \%$ as suggested by thermogravimetric analysis (TGA) and it was calculated by the comparing the char yield from pure carbon, pure alendronate and alendronate loaded carbon at the same temperature and in $\mathrm{N}_{2}$ atmosphere. Alendronate release profile in four different release media is shown in figure 4. Pure alendronate was completely dissolved in less than 30 minutes in the release media of $\mathrm{pH}=1.2$ and hence could not be used for the rest of the media. On the contrary, alendronate from the mesoporous carbons exhibited a controlled release profile and it continued to release after 11 hours of starting the experiment in the $\mathrm{pH}$ of 7.4. In $\mathrm{pH}=1.2$, the mesoporous carbon released only $20 \%$ of total release, followed by about $8-9 \%$ in $\mathrm{pH}=4.5$ and 6.8 . Largest release of about $64 \%$ took place in $\mathrm{pH}=7.4$.

High loading of alendronate in this nitrogen doped mesoporous carbon as well as the largest release of this drug in the medium with highest $\mathrm{pH}$ may be attributed to the hydrogen bonding between alendronate and nitrogen functionalities on the carbon surface. As reported in the previous literature [24], it has been suggested that such hydrogen bonding may form between $-\mathrm{OH}$ or $\mathrm{O}^{-}$groups of alendronate and $\mathrm{N}$ atoms or $\mathrm{NH}^{+}$species present in the carbon surface. (Scheme 1). It can also be hypothesized that normal dispersive forces required for the physisorption of alendronate within the carbon materials may not be strong enough for the significant adsorption of drug and therefore, adsorption was not more than $1-2 \%$ in the relatively higher surface area carbons (not shown in this communication). On the contrary, nitrogen functionalities present in our material enhanced the adsorption more than double that of other carbons. The hypothesis on the hydrogen bonding may also be confirmed from the release pattern of drug. In lower $\mathrm{pH}$, the hydrogen bonding was strong enough to hold the majority of alendronate molecules within the pore surface. At higher $\mathrm{pH}$, such bonding turned weak or almost non-existing and hence majority of the drug molecules was released. The burst release or relatively higher release of alendronate in $\mathrm{pH}=1.2(\sim 20 \%)$ compared to two other $\mathrm{pH}$ (4.5 and 6.8) may be attributed to those alendronate molecules that were present near the pore mouth or not within the influence of hydrogen bonding. Although we did not perform an optimization study on nitrogen content that relates to the trade-off between higher alendronate loading versus controlled release profile, it is clear that very high nitrogen functionalities might have delayed 
the release beyond the actual residence time of the carbon within the gastro-intestinal (GI) tract. As mentioned earlier, side effect of release of alendronate in stomach is quite severe; it causes severe burn and damage in the esophagus and the patients need to follow strict protocol upon taking this medication. As the release amount of alendronate from mesoporous carbon in $\mathrm{pH}=1.2$ (stomach) is quite small compared to rest of the media ( $\mathrm{pH}$ equivalent to that of intestine or colon), it can be argued that such formulation will cause less side effect for oral delivery compared to that of pure drug.

Higuchi kinetics [44] is one of the most common pharmacokinetics equations to model

drug release rates and it is given by $a=k t^{\frac{1}{2}}$, where $a$ is drug release amount, $k$ is Higuchi rate constant and $t$ is the release time. Rate constant $k$ can be calculated by linear regression of $a$ versus $t^{1 / 2}$. For alendronate release from mesoporous carbon, the Higuchi rate constant values were calculated as $0.0267,0.0148,0.0068$, and $0.0488(\mathrm{mg} / \mathrm{g})\left(1 / \mathrm{s}^{1 / 2}\right)$ for $\mathrm{pH}=1.2,4.5,6.8$ and 7.4 , respectively.

\section{Conclusions}

In this phase of research, we have synthesized nitrogen doped mesoporous carbons from resorcinol as carbon precursor, Pluronic F127 as structure dictating agent and dicyandiamide as nitrogen source. The resultant mesoporous carbon has BET surface area $1066 \mathrm{~m}^{2} / \mathrm{g}$ and total pore volume of $0.6 \mathrm{~cm}^{3} / \mathrm{g}$. It has nitrogen content of $0.5 \%$. The particles are of irregular shape and the size varied within 1-200 $\mu \mathrm{m}$. The alendronate loading amount in this carbon is $5 \%$, which is higher than other porous carbon materials with higher surface area. In a release experiment with four different $\mathrm{pH}$ values of 1.2, 4.5, 6.8 and 7.4, representing stomach, duodenum, small intestine and colon, respectively, it exhibited a much sustained release pattern compared to pure alendronate. Unlike pure alendronate that was completely dissolved in less than 30 minutes in $\mathrm{pH}=1.2$, the mesoporous carbon released only $20 \%$ of the drug in this $\mathrm{pH}$ and maximum of amount of drug (64\%) was released in $\mathrm{pH}=7.4$. The higher uptake of alendronate in this nitrogendoped mesoporous carbon and its delayed release may be attributed to the hydrogen bonding between the drug and nitrogen functionalities on the carbon surface. Based on the results, it can be hypothesized that such delayed release may decrease the side effects and strict protocol of 
alendronate use in patients, although that requires much more rigorous studies with human subjects in future.

\section{Acknowledgment}

D.S. acknowledges the faculty development award from School of Engineering (SOE) of Widener University. A.S. acknowledges funding from SOE of Widener University for senior project, which contributed to the part of this study. The authors acknowledge the student undergraduate team members, including Tara Moken, Lauren Benham and Ather Malik for their assistance in the experiment. TEM (J.C.) and SEM (D.K.H.) experiments were conducted at the Center for Nanophase Materials Sciences, which is a DOE Office of Science User Facility.

\section{References}

[1] G.A. Rodan, T.J. Martin, Science 289 (2000) 1508.

[2] S. Colucci, V. Minielli, G. Zambonin, N. Cirulli, G. Mori, M. Serra, V. Patella, A. Zambonin Zallone, M. Grano, Calcif. Tissue Int. 63 (1998) 230.

[3] H. Fleisch, Drugs 42 (1991) 919-944.

[4] E.R. Van Beek, L.H. Cohen, I.M. Leroy, F.H. Ebetino, C.W.G.M. Löwik, S.E. Papapoulos, Bone 33 (2003) 805.

[5] P.D. Miller, Clin. Ther. 27 (2005) 361

[6] A. Goziotis, B. Sukhu, M. Torontali, M. Dowhaniuk, H. C. Tenenbaum. Bone 16 (1995) 317S-327S.

[7] H. C. Tenenbaum, M. Torontali, B. Sukhu. Bone 13 (1992) 249-255.

[8] C. Vitte, H. Fleisch, H. L. Guenther, Endocrinology 137 (1996) 2324-2333.

[9] N. Giuliani, M. Pedrazzoni, G. Negri, G. Passeri, M. Impicciatore, G Girasole, Bone 22 (1998) 455-461.

[10] K. M. Soileau, J Periodontol. 77 (2006) 738-743.

[11] H. L. Wang, D. Weber, L. K. McCauley, J Periodontal 78 (2007) 584-594.

[12] A. Sharma, A.R. Pradeep, J. periodontology 83 (2012) 11-18.

[13] E. Siris, R.S. Weinstein, R. Altman, J.M. Conte, M. Favus, A. Lombardi, K. Lyles, H.

McIlwain, W.A. Murphy Jr., C. Reda, R. Rude, M. Seton, R. Tiegs, D. Thomson, J.R. Tucci, A.J. Yates, M. Zimering, J. Clin. Endocrinol. Meta. 81 (1996) 961.

[14] P.P. Major, A. Lipton, J. Berenson, G. Hortobagyi, Cancer 88 (2000) 6. 
[15] F. Lanza, B. Sahba, H. Schwartz, S. Winograd, J. Torosis, H. Quan, R. Reyes, T. Musliner, A. Daifotis, A. Leung, Am. J. Gastroen. 97 (2002) 58.

[16] R.G.G. Russell, M. J Rogers, Bone 25 (1999) 97.

[17] A. Erza, G. Golomb, Adv. Drug Deliver. Rev. 42 (2000) 175.

[18] F. Lanza, B. Sahba, H. Schwartz, S. Winograd, J. Torosis, H. Quan, R. Reyes, T. Musliner, A. Daifotis, A. Leung, Am. J. Gastroen. 97 (2002) 58.

[19] C. W. Kim, Y. P. Yun, H. J. Lee, Y. S. Hwang, I. K. Kwon, S. C. Lee, J. Controlled Release 147 (2010) 45-53.

[20] Z. Jindong, T. Hai, G. Junchao, W. Bo, B. Li, W. B. Qiang, Orthopedics 33 (2010).

[21] H. Seshima, M. Yoshinari, S. Takemoto, M. Hattori, E. Kawada, T. Inoue, Y. Oda, J Biomed. Mater. Res. B. Appl. Biomater. 78 (2006) 215-221

[22] S. Panzavoltaa, P. Torricellib, B. Braccia, M. Finib, A. Bigia, J. Inorganic Biochemistry 104 (2010) 1099- 1106

[23] D. S. Garbuz, Y. Hu, W. Y. Kim, K. Duan, B. A. Masri, T. R. Oxland, H. Burt, R. Wang, C. P. Duncan, J. Bone Joint Surg. Am. 90 (2008) 1090-1100

[24] F. Balas , M. Manzano , P. Horcajada , M. Vallet-Regí, J. Am. Chem. Soc. 128 (2006) 8116-8117

[25] M. Vallet-Regí, M. Colilla, and I. Izquierdo-Barba, J. Biomed. Nanotechnol. 4 (2008) 1-15

[26] A. Nieto, F. Balas, M. Colilla, M. Manzano, M. Vallet-Regí, Microporous and Mesoporous Materials 116 (2008) 4-13

[27] M. Colilla, I. Izquierdo-Barba, M. Vallet-Regí, Microporous and Mesoporous Materials 135 (2010) 51-59

[28] X. Shi, Y. Wang, L. Ren, Y. Gong, D. A. Wang, Pharmaceutical Research 26 (2009) 422430

[29] E. H. Nafea, M. A. EI-Massik, L. K. EI-Khordagui, M. A. Marei, N. M. Khalafallah, J. Microencapsul. 24 (2007) 525-538.

[30] C. Clementi, K. Miller, A. Mero, R. Satchi-Fainaro, G. Pasut, Mol. Pharmaceutics 8 (2011) 1063-1072

[31] A. Ezra, G. Golomb, Adv. Drug Deliv. 42 (2000) 175-195

[32] H. K. Han, H. J. Shin, D. H. Ha, European Journal of Pharmaceutical Sciences 46 (2012) $500-507$

[33] X. Zhang, T. Zhang, Y. Ye, H. Chen, H. Sun, X. Zhou, Z. Ma, B. Wu, European Journal of Pharmaceutics and Biopharmaceutics 94 (2015) 30-41

[34] J. Zhu, L Liao, X. Bian, J, Kong, P. Yang, B. Liu, Small 8 (2012) 2715-2720.

[35] D. Saha, E. A. Payzant, A. S. Kumbhar, A. K. Naskar, ACS Appl. Mater. Interfaces 5 (2013) 5868-5874.

[36] D. Saha, K. E. Warren, A. K. Naskar, Carbon 71 (2014) 47-57.

[37] X. Wang, P. Liu, Y. Tian, L. Zang, Microporous Mesoporous Mater 145 (2011) 98-103.

[38] D. Saha, K. E. Warren, A. K. Naskar, Microporous Mesoporous Mater 196, (2014) $327-334$. 
[39] D. Saha, T. Moken, J. Chen, D. K. Hensley, K. Delaney, M. A. Hunt, K. Nelson, A. Spurri, L. Benham, R. Brice, M. Azoro, RSC Adv. 5 (2015) 23699

[40] M. F. Gencoglu, A. Spurri, M. Franko, J. Chen, D. K. Hensley, C. L. Heldt, D. Saha, ACS Appl. Mater. Interfaces 6 (2014) 15068.

[41] D. Saha, C.L. Heldt, M. F. Gencoglu, K. S. Vijayaragavan, Jihua Chen, A. Saksule, Toxicology (under review)

[42] J. Wei, D. Zhou, Z. Sun, Y. Deng, Y. Xia, D. Zhao, Adv. Funct. Mater. 23 (2013) 2322

[43] S. Sultana, S. Talegaonkar, G. Mittal, A. Bhatnagar, F. J. Ahmad, Chromatographia 72 (2010) 321-326.

[44] J. Siepmann, N.A. Peppas, Intl J. Pharmaceutics 418 (2011) 6-12.

[45] https://www.wavefun.com/products/spartan.html (accessed March 2016)
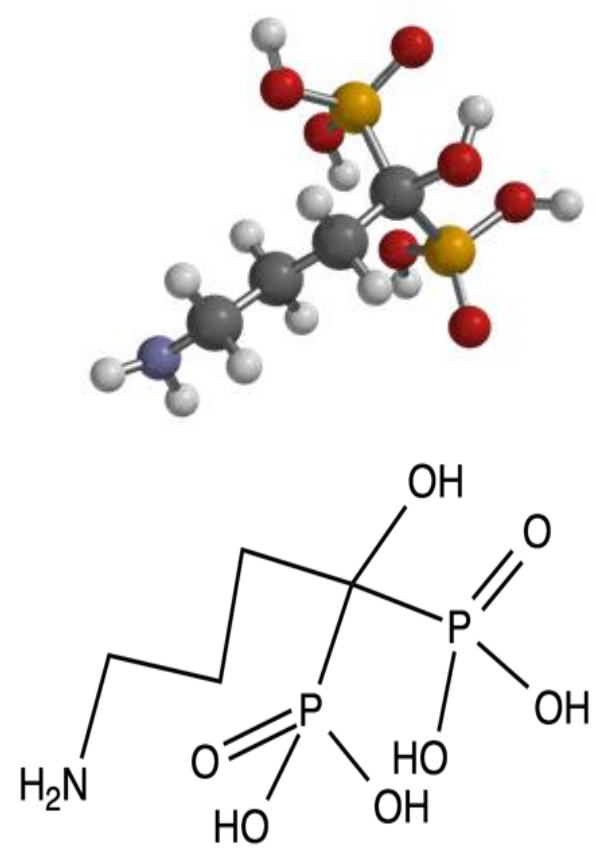

Figure 1. Molecular structure of alendronate, stabilized ball and stick configuration by Spartan software [45], grey: carbon, white: hydrogen, blue: nitrogen, orange: phosphorous, red: oxygen (top), molecular formula (bottom). Colored picture is available in online version only. 


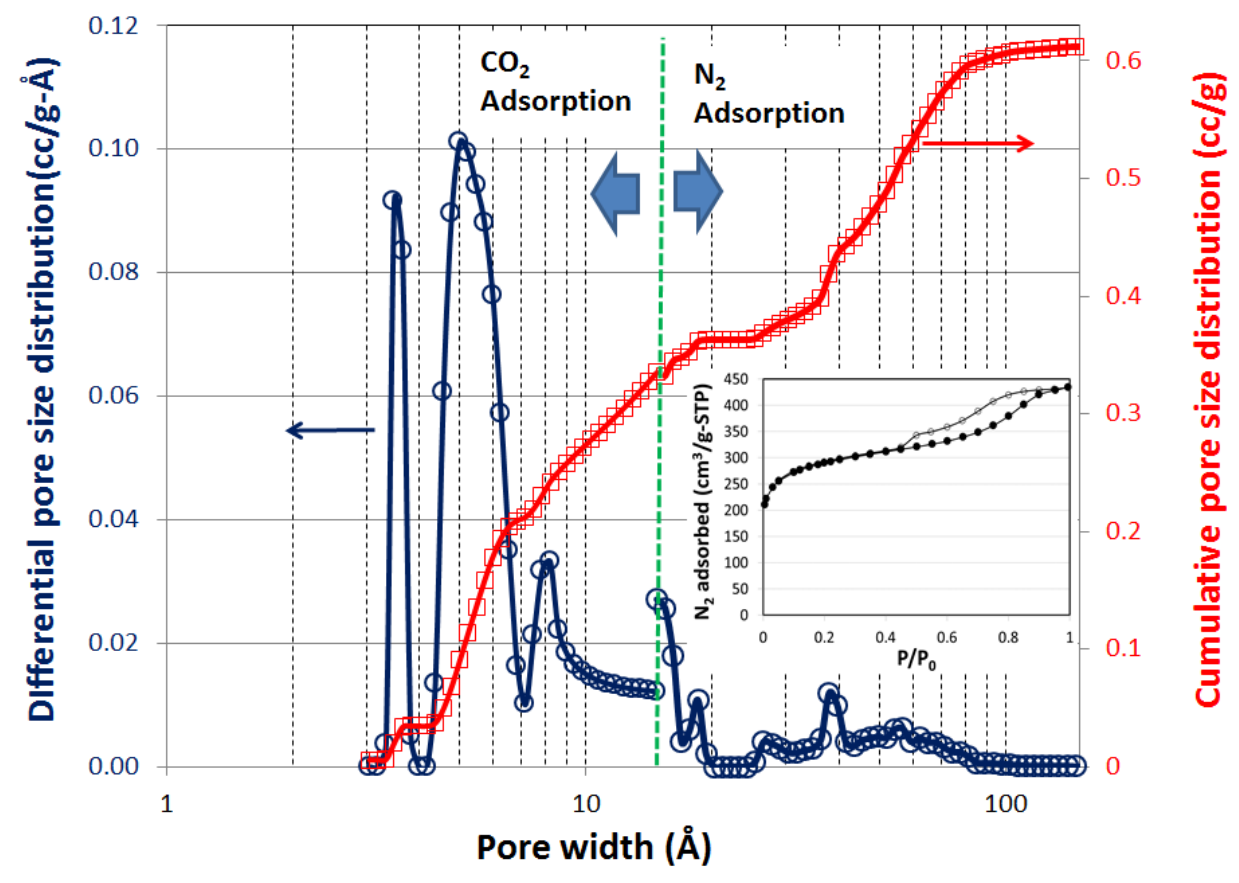

Figure 2. Pore size distribution obtained from $\mathrm{N}_{2}$ and $\mathrm{CO}_{2}$ adsorption plot by non-local density function theory (NLDFT), Nitrogen adsorption-desorption on mesoporous carbons on carbons at $77 \mathrm{~K}$ (inset) 


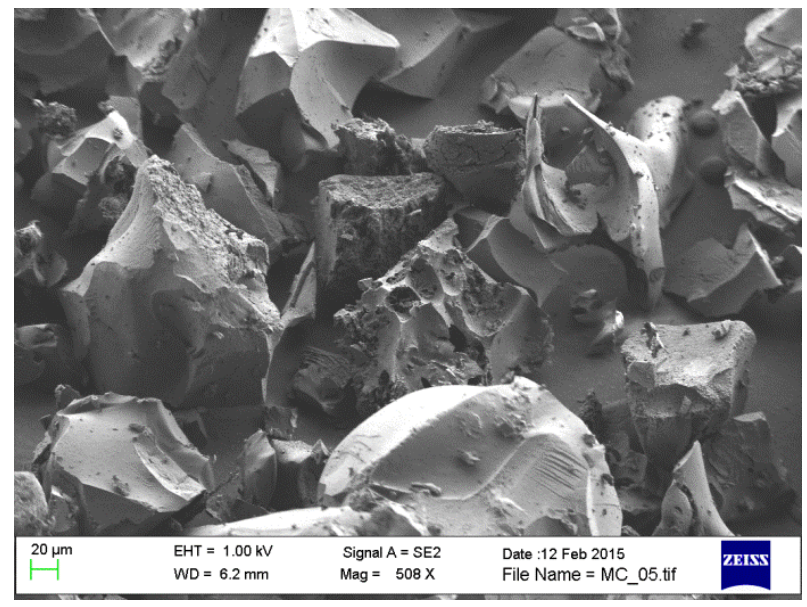

(a)

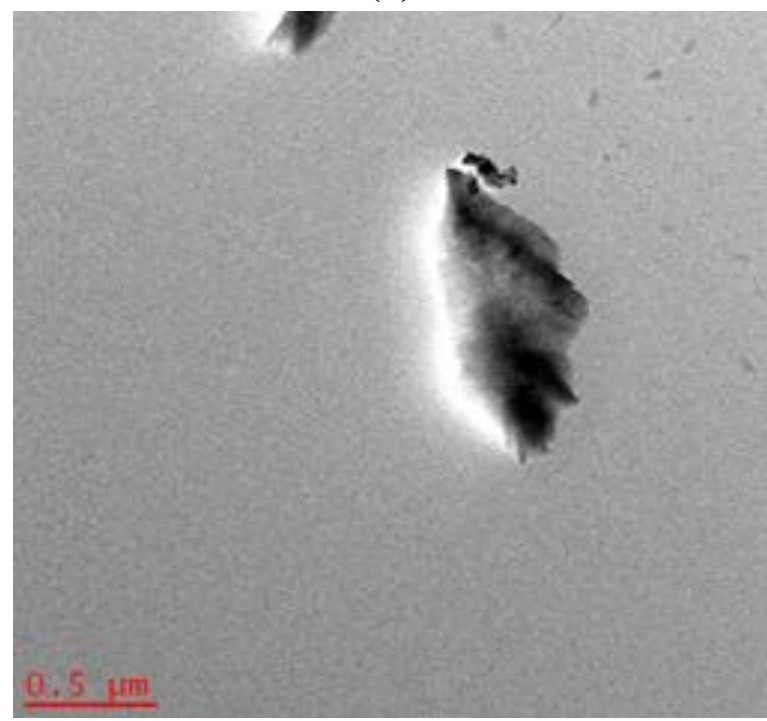

(c)

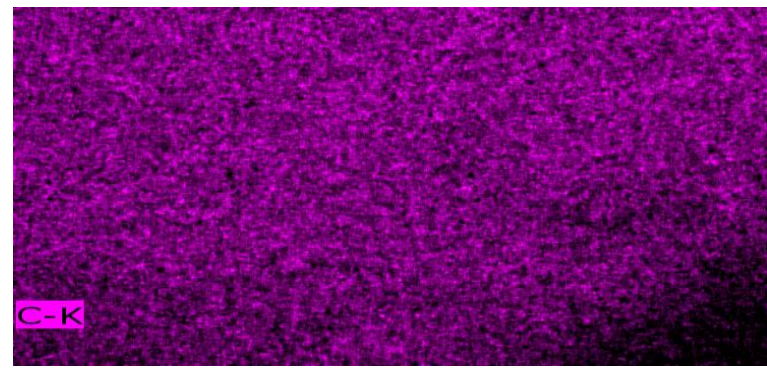

(e)

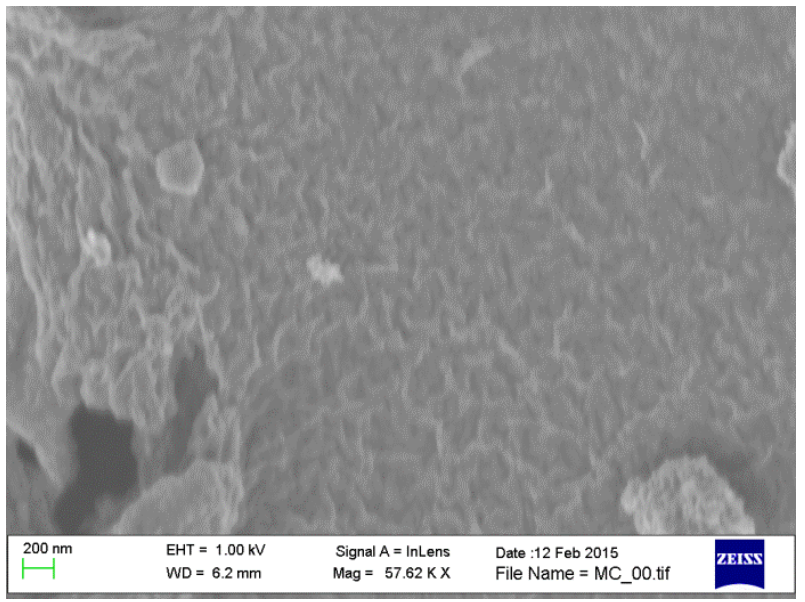

(b)

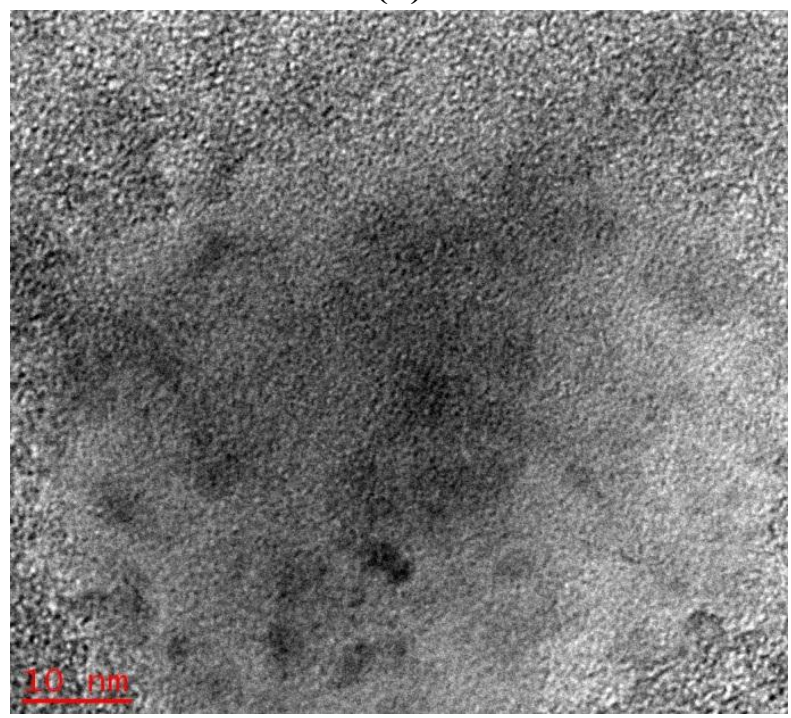

(d)

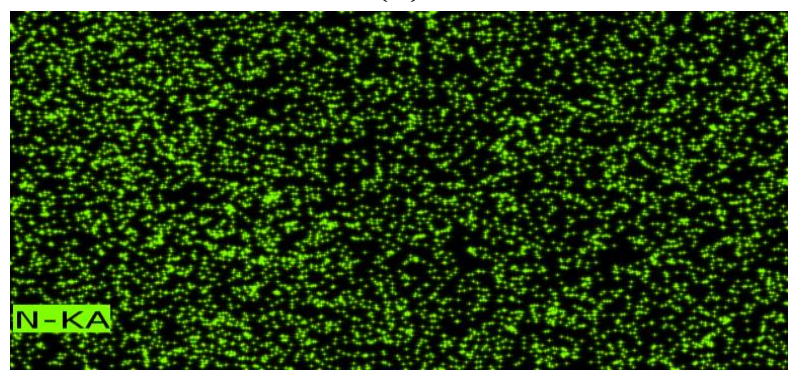

(f)

Figure 3. SEM images in low and high magnification ( $a$ and b), TEM images in low and high magnification (c and d), Energy dispersive results in x-ray (EDX) results for carbon (e) and nitrogen (f) in the mesoporous carbon. Colored picture is available in online version only. 


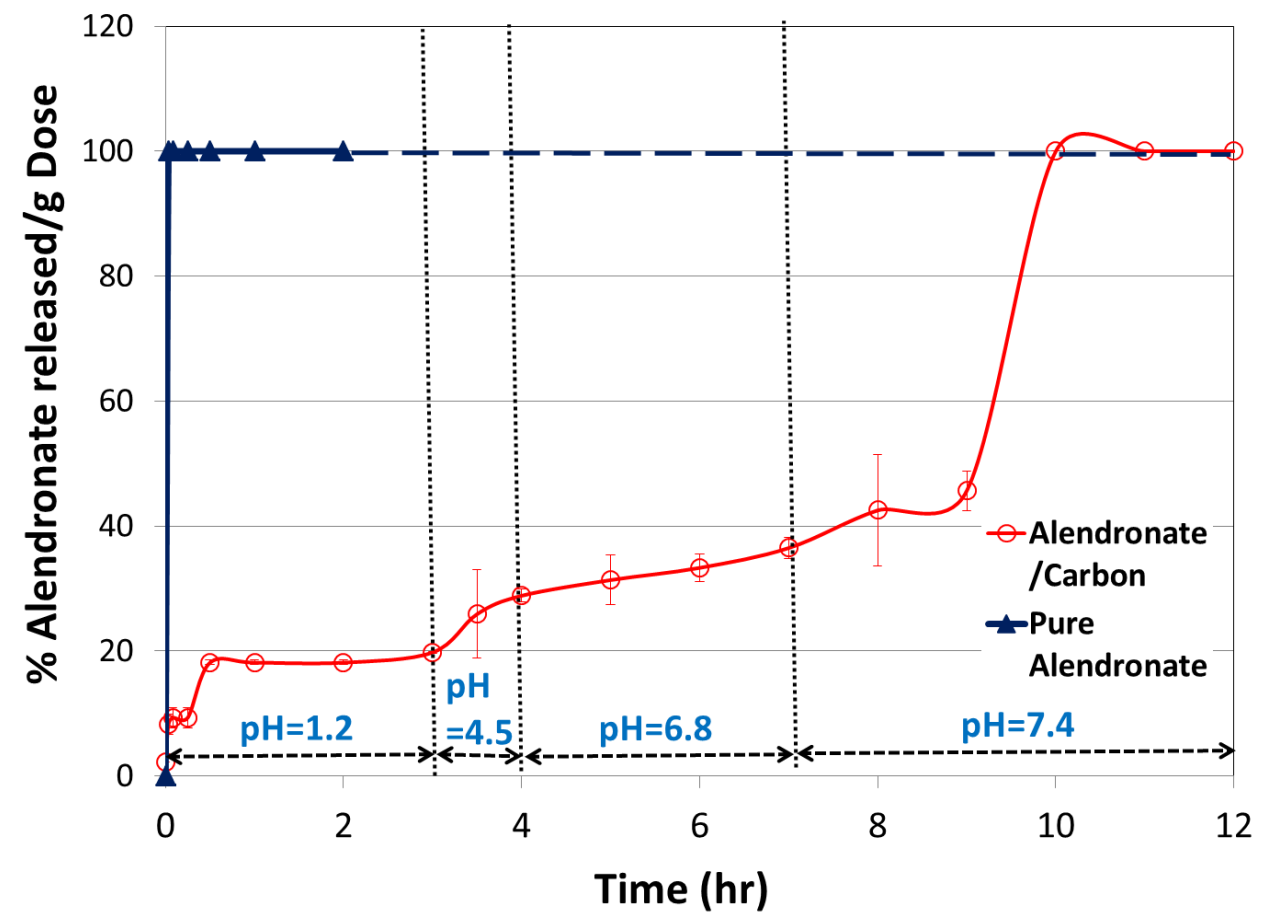

Figure 4. Results of release experiments of alendronate from nitrogen-doped micro-mesoporous carbon in four different $\mathrm{pH}$ media to simulate gastro-intestinal conditions (red). Results were compared with pure alendronate (blue). 


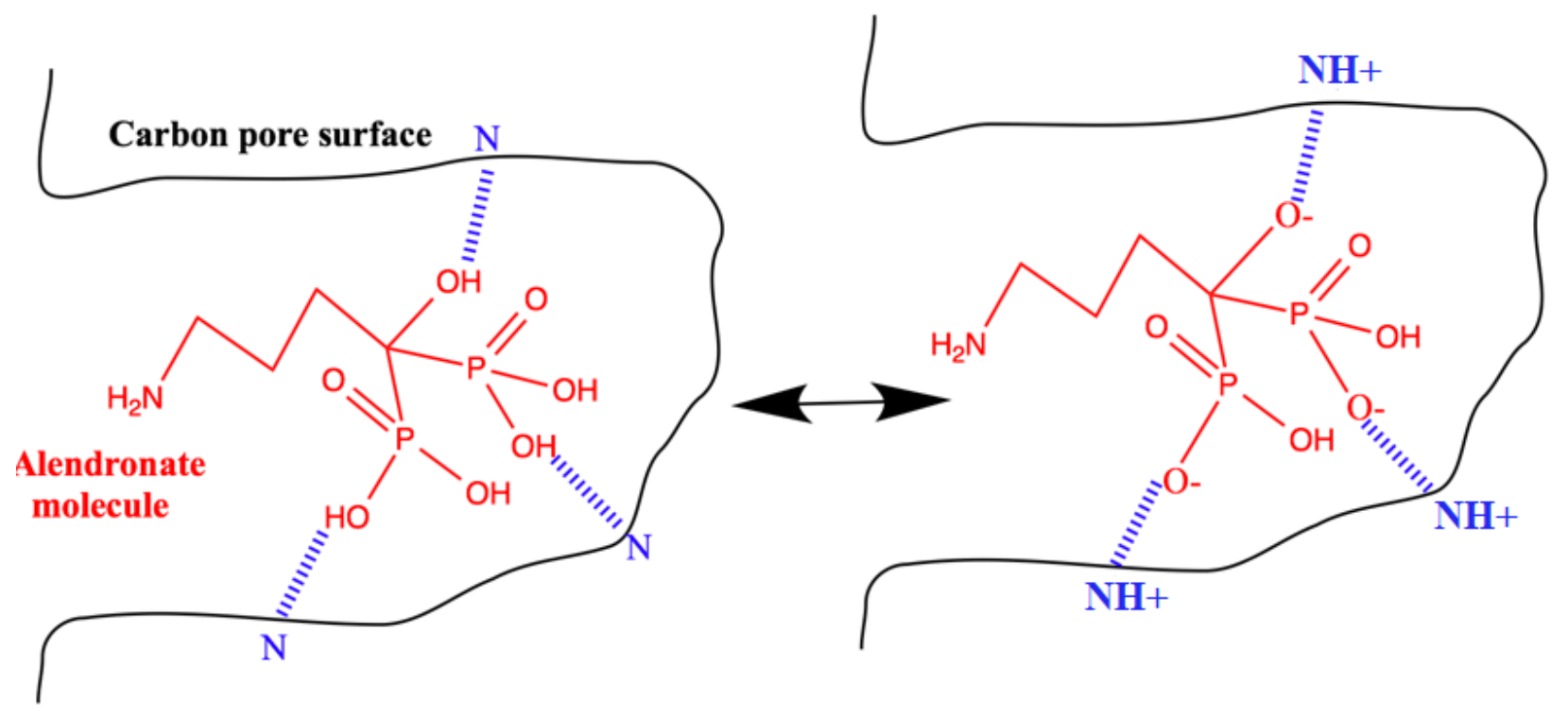

Scheme 1. Hypothetical scheme of adsorbed alendronate within the pore surface of carbon, stabilized by hydrogen bonding with the nitrogen species present on carbon surface 


\section{Graphical Abstract}

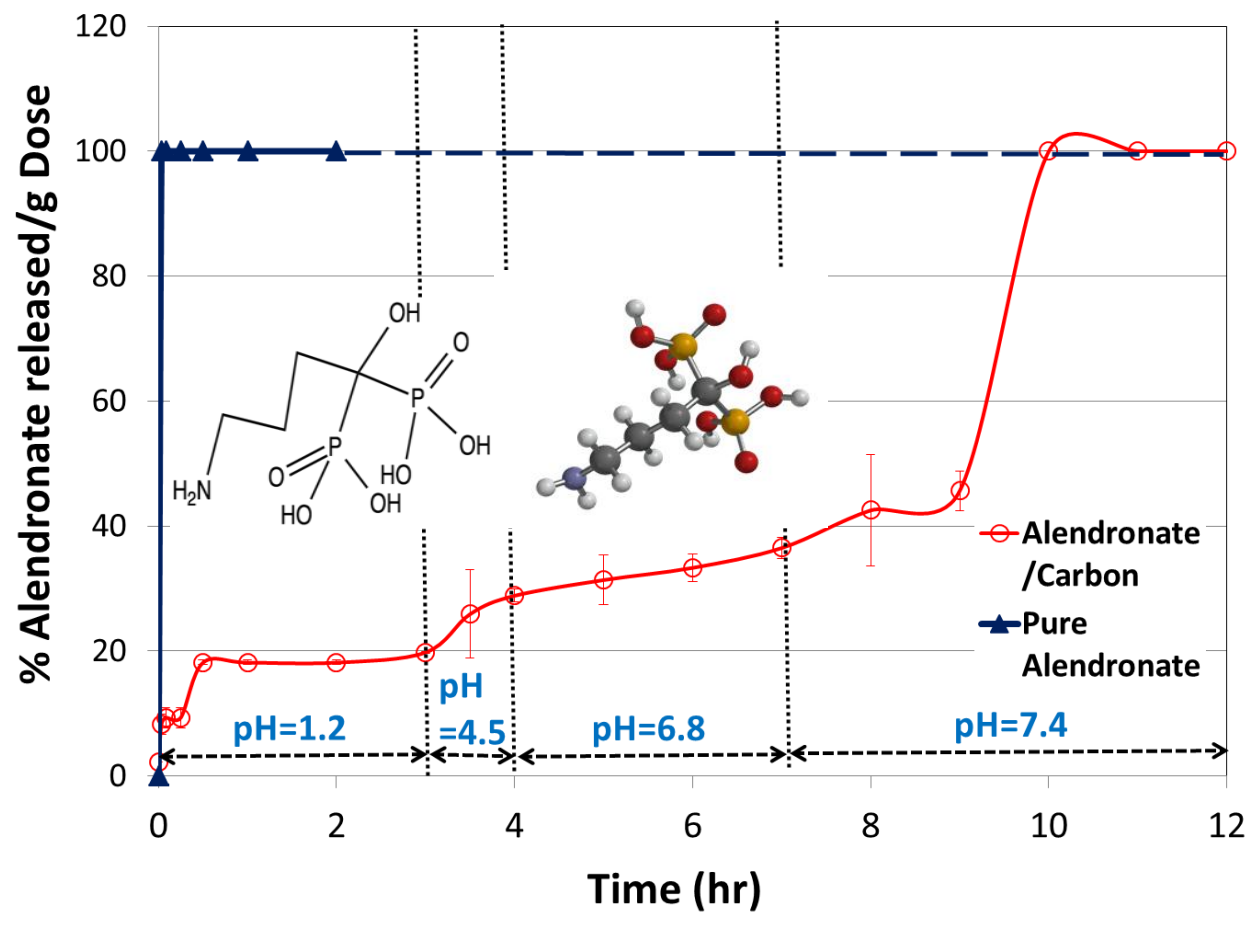

